\title{
Neuron Replating, a Powerful and Versatile Approach to Study Early Aspects of Neuron Differentiation
}

\author{
Felix Schneider, ${ }^{1,2,3}$ Thuy-An Duong, ${ }^{1}$ and ${ }^{-M a r c o}$ B. Rust ${ }^{1,2,3}$
}

https://doi.org/10.1523/ENEURO.0536-20.2021

\begin{abstract}
${ }^{1}$ Molecular Neurobiology Group, Institute of Physiological Chemistry, University of Marburg, Marburg 35032, Germany, ${ }^{2}$ Center for Mind, Brain and Behavior (CMBB), University of Marburg and Justus-Liebig-University Giessen, Marburg 35032, Germany, and ${ }^{3}$ DFG Research Training Group, Membrane Plasticity in Tissue Development and Remodeling, GRK 2213, University of Marburg, Marburg 35032, Germany
\end{abstract}

\begin{abstract}
Neuron differentiation includes formation and outgrowth of neurites that differentiate into axons or dendrites. Directed neurite outgrowth is controlled by growth cones that protrude and retract actin-rich structures to sense environmental cues. These cues control local actin filament dynamics, steer growth cones toward attractants and away from repellents, and navigate neurites through the developing brain. Rodent hippocampal neurons are widely used to study the mechanisms underlying neuron differentiation. Genetic manipulation of isolated neurons including gene inactivation or reporter gene expression can be achieved by classical transfections methods, but these methods are restricted to neurons cultured for several days, after neurite formation or outgrowth. Instead, electroporation allows gene manipulation before seeding. However, reporter gene expression usually takes up to $24 \mathrm{~h}$, and time course of gene inactivation depends on the half live of the targeted mRNA and gene product. Hence, these methods do not allow to study early aspects of neuron differentiation. In the present study, we provide a detailed protocol in which we combined electroporation-based gene manipulation of mouse hippocampal neurons before initial seeding with a replating step after $2 \mathrm{~d}$ in vitro (DIV) that resets neurons into an undifferentiated stage. By categorizing neurons according to their differentiation stage, thorough morphometric analyses, live imaging of actin dynamics in growth cones as well as guidance cue-mediated growth cone morphologic changes, we demonstrate that differentiation and function of replated neurons did not differ from non-replated neurons. In summary, we provide a protocol that allows to thoroughly characterize differentiation of mouse primary hippocampal neurons.
\end{abstract}

Key words: actin; cofilin; growth; neurite; neuron replating; replating

\section{Significance Statement}

Unraveling the molecular mechanisms that control neuron differentiation requires reporter gene expression or gene inactivation. In mouse primary hippocampal neurons, a widely used cellular system to study neuron differentiation, classical transfection methods are restricted to later stages of differentiation. Instead, electroporation allows genetic manipulation before seeding. However, time course of reporter gene expression or gene inactivation frequently hinders a full characterization of neuron differentiation, specifically of early stages. To circumvent this limitation, we combined electroporation-based genetic manipulation before initial seeding with a replating step after 2 $\mathrm{d}$ in vitro (DIV), which reset neurons into an undifferentiated stage. We show that replated neurons differentiated similar to non-replated neurons. We provide a detailed protocol that allows to comprehensively characterize the molecular mechanisms underlying neuron differentiation. 


\section{Introduction}

During differentiation, neurons undergo striking morphologic changes from spheres to polar cells possessing an axon and a highly branched dendritic compartment (Dotti et al., 1988; da Silva and Dotti, 2002). Essential steps during early neuron differentiation include the formation and outgrowth of neurites, which later differentiate into axons or dendrites. Directed neurite outgrowth depends on growth cones, structures at neurite tips enriched in actin filaments (F-actin) that steer neurites toward attractants and away from repellent cues and, hence, navigate neurites through the developing brain (Gomez and Letourneau, 2014). Cultured hippocampal neurons isolated from mice or rats are widely used cellular systems to study neuron differentiation as they readily polarize on a two-dimensional substrate at very low densities (Dotti et al., 1988; da Silva and Dotti, 2002). Genetic manipulation including gene silencing, gene deletion or reporter gene expression provide powerful approaches to study virtually all biological processes in cellular systems, including neuron differentiation. Electroporation-based nucleofection as well as classical transfection procedures such as liposome-based transfection or calcium phosphate precipitation are the most commonly applied methods for gene transfer into cultured hippocampal neurons as they are far less labor-intensive when compared with virus infection (Dudek et al., 2001; Ohki et al., 2001; Zeitelhofer et al., 2009; Viesselmann et al., 2011; Sun et al., 2013). Unfortunately, efficiency of classical transfection procedures is rather low and these approaches are convenient only for hippocampal neurons cultured for several days, e.g., at around $6 \mathrm{~d}$ in vitro (DIV) or later. Instead, nucleofection allows genetic manipulation of hippocampal neurons before seeding. However, expression of reporter genes usually takes up to $24 \mathrm{~h}$, and more importantly, time course and efficiency of gene silencing or gene deletion depends on the half live of the targeted mRNA and gene product. Consequently, nucleofection of hippocampal neurons does not allow a thorough analysis of neuron differentiation, specifically not of early processes during neuron differentiation. Thus, experimental approaches are needed to circumvent these limitations. We here report a protocol to reset primary hippocampal neurons from embryonic mice at DIV2 into an undifferentiated stage. Before initial seeding, these neurons can be

This work was supported by the Deutsche Forschungsgemeinschaft (DFG) Research Grant RU 1232/7-1 (to M.B.R.). F.S. was supported by the DFG Research Training Group Membrane Plasticity in Tissue Development and Remodeling Grant GRK 2213.

Acknowledgements: We thank Renate Gondrum for excellent technical support, Dr. Robert Grosse (University of Freiburg, Germany) for GFP-actin and LifeAct-GFP constructs, Dr. David Solecki (St. Jude Children's Research Hospital Memphis) for mCherry-Cre constructs, and Dr. Walter Witke (University of Bonn, Germany) for $\mathrm{ADF}^{-/-} / \mathrm{Cfl}^{\mathrm{fl} / \mathrm{fl} \mathrm{x}}$ mice.

Correspondence should be addressed to Marco B. Rust at rust@unimarburg.de.

https://doi.org/10.1523/ENEURO.0536-20.2021

Copyright @ 2021 Schneider et al.

This is an open-access article distributed under the terms of the Creative Commons Attribution 4.0 International license, which permits unrestricted use, distribution and reproduction in any medium provided that the original work is properly attributed. manipulated genetically by means of nucleofection. We show that a combination of nucleofection and replating allows to study early aspects of neuron differentiation.

\section{Materials and Methods}

\section{Mice}

Generation of ADF-/-/Cfl $1^{\text {flx/flx }}$ mice has been reported before (Bellenchi et al., 2007; Wolf et al., 2015; Zimmermann et al., 2015). Mice were housed with food and water available ad libitum on 12/12 h light/dark cycles. Treatment of mice was in accordance with the German law for conducting animal experiments and followed the guidelines for the care and use of laboratory animals of the National Institutes of Health. Killing of mice has been approved by internal animal welfare authorities (references: AK-5-2014, AK-6-2014, AK-122020). Genetic inactivation of $\mathrm{Cfl} 1$ in neurons from ADF-/-/Cfl1 $1^{\mathrm{fl} / \mathrm{fl} \times \mathrm{x}}$ mice was achieved by nucleofection of catalytic active mCherry-Cre. ADF-/-/Cfl $1^{\text {flx/flx }}$ neurons expressing a mutant, catalytic inactive mCherryCre served as controls. Both constructs have been achieved from the Solecki lab (Kullmann et al., 2020).

\section{Hippocampus dissection and neuron isolation}

One day before neuron isolation, glass cover slips (13 $\mathrm{mm}$ in diameter, VWR) were placed into 24-well plates and coated overnight with $0.1 \mathrm{mg} / \mathrm{ml}$ poly-L-lysine hydrobromid (dilution of $1 \mathrm{mg} / \mathrm{ml}$ poly-L-lysine with $0.1 \mathrm{~m}$ boric acid at $\mathrm{pH} 8.5$ ) in a humidified incubator at $37^{\circ} \mathrm{C}$ and $5 \%$ $\mathrm{CO}_{2}$. For replating, 24-well plates without cover slips were coated with $0.05 \mathrm{mg} / \mathrm{ml}$ poly-L-lysine hydrobromid and similar incubated as above. On the day of neuron isolation, plates were washed twice with $\mathrm{ddH}_{2} \mathrm{O}$ and equilibrated either with 500- $\mu$ l nucleofection medium (DMEM31966; Invitrogen) supplemented with $10 \%$ fetal bovine serum (FBS; Invitrogen) or for non-nucleofected neurons with neurobasal (NB; Invitrogen) medium. Mice of either sex were killed at embryonic day (E)18.5 by decapitation, and brains were dissected on ice in Leibovitz's L15Medium with $7 \mathrm{~mm}$ HEPES (L15+H, Invitrogen). After removal of the meninges, hippocampi of each embryo were isolated and collected in a tube containing cooled $\mathrm{L} 15+\mathrm{H}$. Thereafter, medium was replaced by $500-\mu \mathrm{l}$ prewarmed TrypLE Express (Invitrogen) per embryo and incubated for $6 \mathrm{~min}$ at $37^{\circ} \mathrm{C}$. Subsequently, hippocampi were washed twice with NB medium containing 2\% B27, $2 \mathrm{~mm}$ GlutaMax, $100 \mu \mathrm{g} / \mathrm{ml}$ streptomycin, and $100 \mathrm{U} / \mathrm{ml}$ penicillin (NB+, Invitrogen). After washing, neurons were triturated in $1 \mathrm{ml} \mathrm{NB}+$ by pipetting seven times up and down with a P1000 pipette. Neuron solution was filled up to $1 \mathrm{ml} \mathrm{NB}+$ medium per embryo and density was calculated by using a hemocytometer. Thereafter, neurons were plated at a density of 60,000 cells per well. $5 \mathrm{~h}$ after plating, medium was completely replaced by NB+ medium.

\section{Electroporation of hippocampal neurons}

In some experiments, neurons were electroporated before plating. In these experiments, electroporation was 
performed according to manufacturer's protocol by using the Amaxa P3 Primary Cell 4D-Nucleofector $X$ kit $L$ (Lonza) and 4D-Nucleofector (Lonza). For nucleofection, 250,000 neurons were transfected with $3-\mu \mathrm{g}$ plasmid and the entire neuron suspension was plated in a single well of a 24-well plate in nucleofection medium; $5 \mathrm{~h}$ after plating, medium was completely replaced by $\mathrm{NB}+$ medium.

\section{Replating of hippocampal neurons}

At DIV2, neurons were detached and plated again (replated) on cover slips. Before replating, coverslips were prepared as described above. For replating, condition medium $(350-\mu \mathrm{l}$ medium from each well $+200 \mu \mathrm{l}$ fresh $\mathrm{NB}+$ medium for each well) was collected and kept in the water bath at $37^{\circ} \mathrm{C}$. Remaining medium was aspirated, replaced with prewarmed $500-\mu l$ TrypLE Express per well and incubated for $15 \mathrm{~min}$ in the humidified incubator. To detach the cells after incubation, the bottom of the well was rinsed twice with the TrypLE Express, and 500- $\mu$ l prewarmed NB+ medium was added to stop enzymatic reaction. Again, the bottom of the well was rinsed twice with the medium-enzyme solution and then completely transferred in to 1.5-ml cups and centrifuged for $5 \mathrm{~min}$ with $7000 \mathrm{rpm}$. Thereafter, pelleted neurons were re-suspended in 500- $\mu$ l condition medium and plated on cover slips in 24-well plates and incubated at $37^{\circ} \%$ with $5 \%$ $\mathrm{CO}_{2}$ until further processing.

\section{Immunocytochemistry}

One or $2 \mathrm{~d}$ after seeding or replating, neurons were fixed for $10 \mathrm{~min}$ in $4 \%$ paraformaldehyde in PBS under cytoskeleton preserving conditions ( $\mathrm{pH} 7-7.5)$. After washing with PBS, neurons were incubated with $0.4 \%$ gelatin with $0.5 \%$ Triton $\mathrm{X}-100$ in PBS (carrier solution) for $5 \mathrm{~min}$, followed by incubation with the primary antibody rabbit anti-Dcx (1:500, Abcam; in carrier solution). After $90 \mathrm{~min}$ incubation, neurons were washed with PBS and incubated with Alexa Fluor 488-coupled phalloidin (1:100, ThermoFisher Scientific) to visualize F-actin and the secondary antibody anti-rabbit IgG coupled to Alexa Fluor 546 (1:500, Invitrogen; in carrier solution). After $60 \mathrm{~min}$ of incubation, neurons were washed with PBS and nuclei were stained with the DNA dye Hoechst (1:1000 in PBS, Invitrogen). Neurons were imaged with a Leica TCS SP5 II confocal microscope setup.

\section{Live cell imaging}

For live cell imaging, neurons were seeded either directly after nucleofection or after replating in a poly-L-lysine hydrobromid-coated 22-mm glass-bottom dish and cultured for 1d. To measure actin turnover via fluorescence recovery after photobleaching (FRAP), neurons were transfected with GFP-actin (Robert Grosse lab) and imaged with a Leica TCS SP5 II in a chamber heated to $35^{\circ} \mathrm{C}$. For imaging, neurons were washed once and then imaged in $\mathrm{CO}_{2}$-saturated HBS solution (Invitrogen), supplemented with $4.16 \mathrm{~mm} \mathrm{NaHCO}_{3}$ and $2 \mathrm{~mm} \mathrm{CaCl}_{2}$. For prebleaching condition, five images of growth cones were acquired and in total 65 images over a time course of
5 min during fluorescence recovery. Images were analyzed with ImageJ (Schindelin et al., 2012) and recovery curve and parameters were calculated with $\mathrm{R}$. To assess retrograde F-actin flow of growth cones neurons were transfected with LifeAct-GFP (Robert Grosse lab) and imaged in a $\mathrm{CO}_{2}$-regulated chamber maintained at $37^{\circ} \mathrm{C}$. Image acquisition was done with a Leica DMi8 Thunder microscope system and a Leica DFC9000 GTC camera, which acquired images every $5 \mathrm{~s}$ for $5 \mathrm{~min}$. Kymograph generation and analysis was performed with ImageJ (Schindelin et al., 2012).

\section{Growth cone collapse assay and BDNF treatment}

Neurons were treated for $60 \mathrm{~min}$ with $100 \mathrm{ng} / \mathrm{ml}$ BDNF (PeproTech), $1 \mu \mathrm{g} / \mu \mathrm{l}$ Ephrin A5 (R\&D Systems) or $1 \mu \mathrm{g} / \mu \mathrm{l}$ Slit-1 (R\&D Systems) before fixation. Images were acquired with a Leica TCS SP5 II microscope system and analyses were done with ImageJ (Schindelin et al., 2012). Growth cone size was measured for determining BDNF effects, whereas repellent cues treated growth cones were categorized into collapsed and non-collapsed according to previous studies (Müller et al., 1990).

\section{Statistics}

Statistical tests were done in R or Sigma Plot. For comparing mean values between groups, Student's $t$ test or Mann-Whitney $U$ test was performed. Analyzing the rescue conditions, ANOVA with post hoc test was used. Stage distribution and non-collapsed versus collapsed growth cones were tested for differences with $\chi^{2}$ test.

\section{Results}

\section{Replating does not alter hippocampal neuron morphology}

This study aimed at testing whether a combination of nucleofection and replating is a useful approach to study early aspects of hippocampal neuron differentiation. To do so, we isolated hippocampal neurons from C57BI/6 mice at E18.5. Upon nucleofection, hippocampal neurons were seeded in 24-well plates and incubated at standard conditions (Fig. 1). After DIV2, we detached neurons by means of an enzymatic digest and mechanical treatment to reset them into an undifferentiated stage. Thereafter, hippocampal neurons were plated on cover slips and kept in culture, similar to non-replated neurons. To test whether this procedure affected neuron differentiation, we compared neurons 1 or $2 \mathrm{~d}$ after replating (DAR) with non-replated neurons at DIV1 or DIV2, respectively. We stained neurons with the F-actin marker phalloidin and an antibody against doublecortin (Dcx) that labeled neurites (Fig. 2A). This approach allowed us to categorize neurons according to their differentiation stage (Fig. 2B; Dotti et al., 1988). As expected, only a few non-replated DIV1 neurons remained in stage 1, i.e., they formed Factin-enriched lamellipodia, but not yet neurites (Fig. $2 C$ ). The majority developed neurites, but not yet an axon and were assigned to stage 2, while a few neurons already possessed an axon and reached stage 3 (stage 1: $9.48 \pm 2.55 \%$; stage 2: $79.95 \pm 4.43 \%$, stage 3 : 


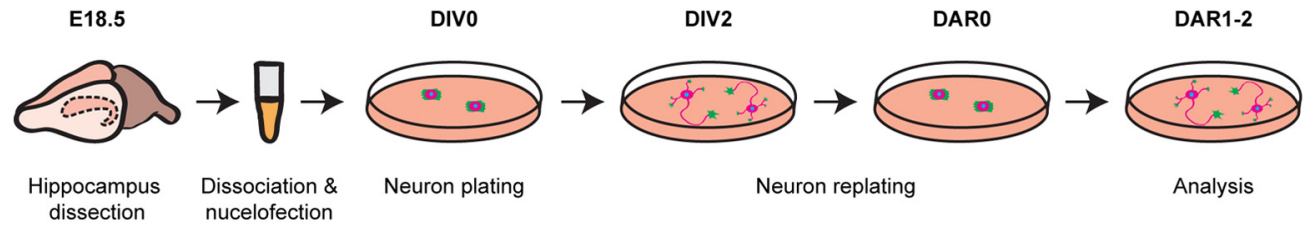

Figure 1. Scheme showing experimental procedure. Timeline and workflow of experimental approach including (1) isolation of hippocampal neurons from E18.5 mice; (2) nucleofection-based genetic manipulation before seeding that could be either reporter gene expression or gene inactivation; (3) culture of hippocampal neurons for $2 \mathrm{~d}$; (4) replating of hippocampal neurons at DIV2 to reset them into an undifferentiated stage; (5) culture of replated neurons until further analyses.

$10.56 \pm 2.83 \%, n>180$ cells from three independent experiments). Very similar to non-replated DIV1 neurons, we found a few neurons in stage 1 and stage 3 at DAR1, while the majority were assigned to stage 2 (stage 1 : $13.05 \pm 2.02 \%$; stage 2: $77.59 \pm 2.90 \%$, stage 3: 9.36 $\pm 2.25 \%, n>340 / 3)$. Comparison between DIV1 and DAR1 cultures revealed no difference in stage distribution $(p=0.44)$. At DIV2, the fraction of non-replated stage 3 neurons increased to roughly one third, and almost all other neurons were in stage 2 (stage 1: $4.81 \pm 2.22 \%$, stage 2: $57.39 \pm 4.17 \%$, stage 3: $37.80 \pm 3.10 \%$; $n>$ 160/3). We found a similar stage distribution among DAR2 neurons (stage 1: $5.32 \pm 1.59 \%$, stage 2: $56.97 \pm$ $3.71 \%$, stage 3: $37.71 \pm 4.56 \% ; n>240 / 3$ ), with no difference when compared with DIV2 cultures $(p=0.81)$.

Antibody staining further allowed us to determine neuron morphology by counting the numbers of primary neurites and neurite endpoints and by calculating the ratio of primary neurites and neurite endpoints as a readout for neuron complexity. We determined these parameters in stage 2 neurons at DAR1 and DAR2 and compared them to non-replated neurons at DIV1 and DIV2, respectively. In DAR1 neurons, the numbers of primary neurites and neurite endpoints was not different from DIV1 neurons (neurites: DIV1: $5.11 \pm 0.38$, DAR1: $4.83 \pm 0.25, p=0.54$; endpoints: DIV1: $5.50 \pm 0.39$, DAR1: $5.90 \pm 0.33, p=$ 0.44 ; Fig. $2 D, E$ ). Instead, the neurite/endpoint ratio was slightly increased by roughly $10 \%$ in DAR1 neurons (DIV1: $1.10 \pm 0.05$, DAR1: $1.24 \pm 0.05, p<0.05 ; n>20$ / 3; Fig. 2F). Compared with DIV2 neurons, the neurite and endpoint numbers were slightly reduced by $8 \%$ and $30 \%$, respectively, in DAR2 neurons (neurites: DIV2: $4.49 \pm 0.26$, DAR2: $4.12 \pm 0.28, p<0.05$; endpoints: DIV2: $7.67 \pm 0.70$, DAR2: $5.38 \pm 0.41, p<0.01$; $n>20 / 3$; Fig. $2 D, E$ ). However, neuron complexity was similar to DIV2 neurons in DAR2 neurons (DIV2: $1.55 \pm 0.11$, DAR2: $1.33 \pm 0.09, p=0.12$; Fig. $2 F$ ). Together, stage distribution did not differ between DAR1 and DIV1 cultures or between DAR2 and DIV2 cultures. Likewise, gross morphology of DAR1 and DAR2 neurons was similar to DIV1 and DIV2 neurons, respectively, and DAR2 neurons showed only minor changes in morphology.

\section{Replating does not alter growth cone size or morphology}

Next, we tested whether replating altered the morphology or function of growth cones, which are relevant for directed neurite outgrowth and neurite navigation through the developing brain. First, we exploited phalloidin-labeled neurons to determine growth cone size and morphology (Fig. 3A). For better comparison, we restricted this analysis to stage 2 neurons. In DIV1 and DIV2 neurons, growth cones size reached roughly 20 or $30 \mu \mathrm{m}^{2}$, respectively (DIV1: $23.05 \pm 1.74 \mu \mathrm{m}^{2}, n>70 / 3$; DIV2: $30.86 \pm 2.25 \mu \mathrm{m}^{2}, n>70 / 3$; Fig. $3 B$ ). Growth cone size did not differ from non-replated DIV1 or DIV2 neurons in neurons from DAR1 or DAR2 cultures, respectively (DAR1: $20.33 \pm 1.00 \mu \mathrm{m}^{2}, n>100 / 3, p=0.18$; DAR2: $\left.29.97 \pm 1.95 \mu \mathrm{m}^{2}, n>100 / 3, p=0.76\right)$. Growth cone morphology was assessed by determining growth cone circularity (area divided by perimeter) and solidity (growth cone area divided by hull area), similar to previous studies (Chitsaz et al., 2015; Dos-Santos Carvalho et al., 2020). Both parameters were not different between growth cones from DAR1 and DIV1 neurons (solidity: DIV1: $0.63 \pm 0.02, n>70 / 3$, DAR1: $0.60 \pm 0.01$, $n>90 / 3, p=0.20$; circularity: DIV1: $0.22 \pm 0.02, n>70 /$ 3, DAR1: $0.25 \pm 0.01, n>90 / 3, p=0.33$; Fig. $3 C$ ). Together, replating neither affected growth cone size nor morphology.

\section{Replating does not alter actin dynamics in growth cones}

Next, as functional readouts, we assessed actin dynamics in replated neurons. We electroporated neurons before seeding to express GFP-actin that allowed us to determine actin turnover in growth cones by FRAP, similar to previous studies (Flynn et al., 2012). We performed FRAP experiments in growth cones from DAR1 neurons and compared actin turnover to growth cones from nonreplated DIV1 neurons. In growth cones from DIV1 neurons, GFP-actin rapidly recovered with a mean half-recovery time $\left(t_{1 / 2}\right)$ of $77.36 \pm 12.29$ s $(n>20 / 3$; Fig. $4 A-C$; Movie 1). We noted a similar GFP-actin recovery in growth cones from DAR1 neurons, with no difference in $t_{1 / 2}(74.04 \pm 10.00 \mathrm{~s}, n>20 / 3, p=0.83$; Fig. $4 A-C$; Movie 2). Further, we calculated the stable actin fraction that did not recover within the time frame of $300 \mathrm{~s}$. This fraction was not different between growth cones from DIV1 and DAR1 neurons (DIV1: $0.78 \pm 0.03$, DAR1: $0.75 \pm 0.03, p=0.500$; Fig. $4 D$ ). Additionally, we electroporated neurons before plating to express LifeAct-GFP, which allowed us to visualize F-actin in living neurons (Riedl et al., 2008; Flynn et al., 2012). Factin appeared similarly dynamic in growth cones from 
A
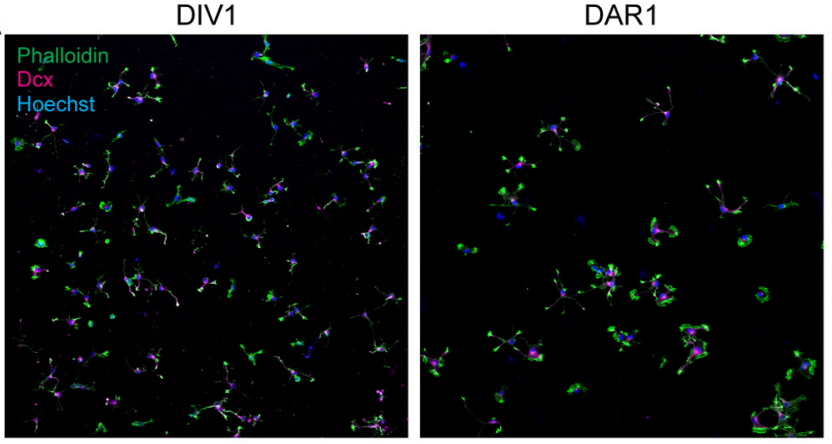

B.
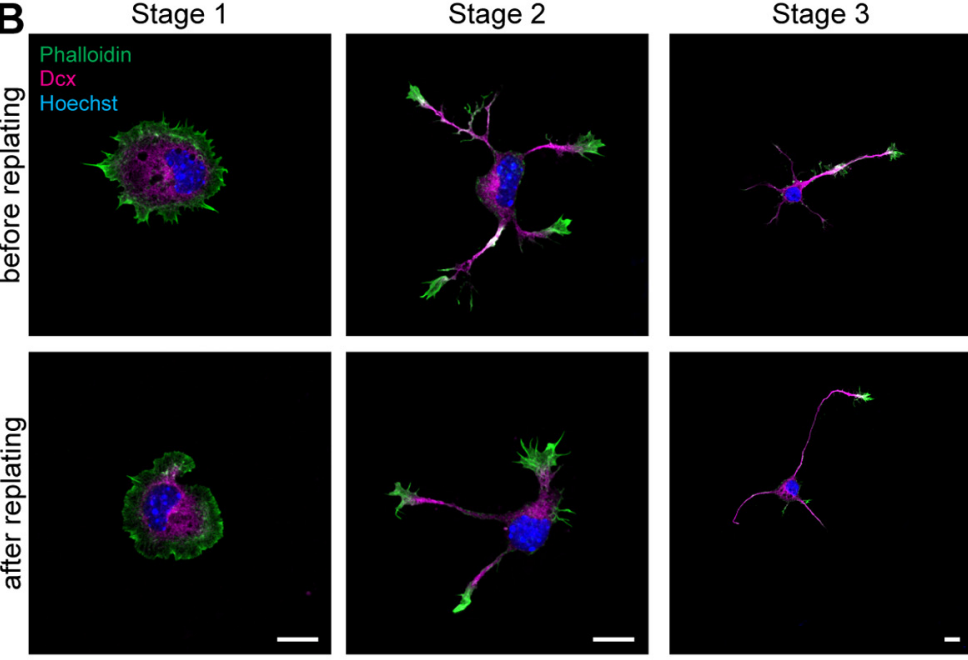

D

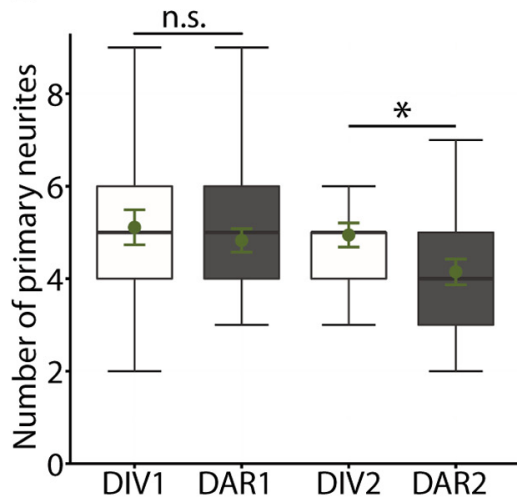

E

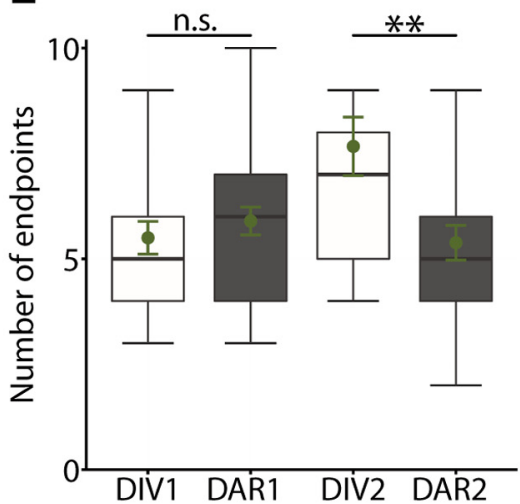

DIV2

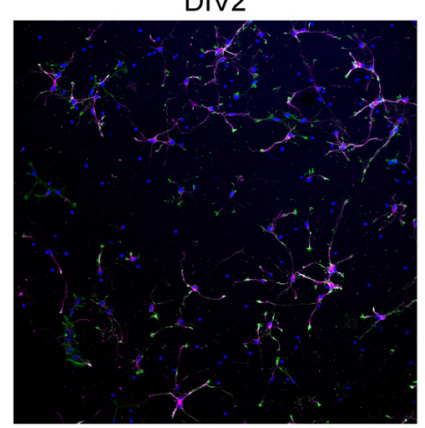

DAR2

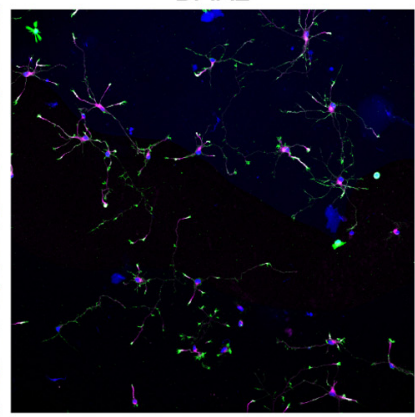

C

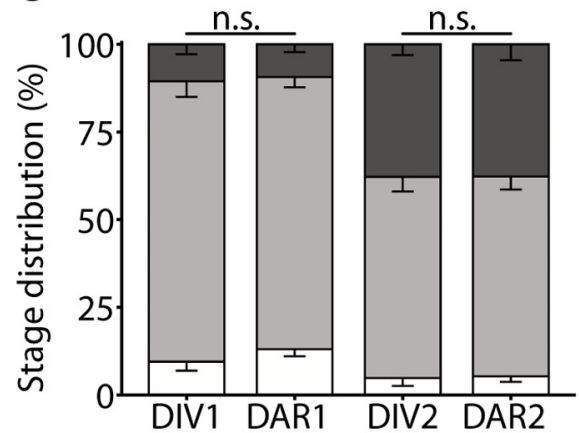

stage 3

stage 2

stage 1

\section{$\mathbf{F}$}

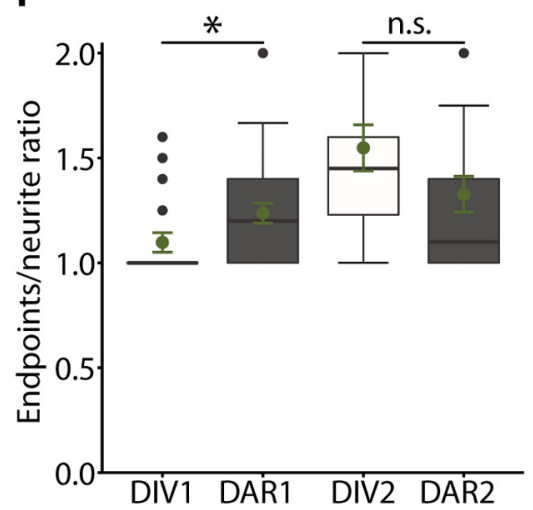

Figure 2. Replating neither alters differentiation nor gross morphology of hippocampal neurons. $\boldsymbol{A}$, Representative micrographs of mouse non-replated hippocampal neurons at DIV1 and DIV2 as well as replated neurons at DAR1 and DAR2. Neurons were stained with the F-actin marker phalloidin (green), with an antibody against Dcx (magenta) and the intercalating dye Hoechst (blue). $\boldsymbol{B}$, Representative micrographs of non-replated and replated stage 1, stage 2, and stage 3 neurons that have been used for morphometric analyses. $\boldsymbol{C}$. Stage distribution of non-replated and replated neurons. Graphs showing $(\boldsymbol{D})$ numbers of primary neurites, $(\boldsymbol{E})$ numbers of neurite endpoints as well as $(\boldsymbol{F})$ primary neurite/neurite endpoint ratio in nonreplated and replated neurons. Scale bars: $50 \mu \mathrm{m}(\boldsymbol{A})$ and $10 \mu \mathrm{m}(\boldsymbol{B})$; ns: $p>0.05,{ }^{*} p<0.05,{ }^{* *} p<0.01$. Green dots indicate mean values with SEM.

DAR1 and DIV1 neurons (Movies 3, 4). Indeed, kymograph analysis revealed similar average retrograde flow velocity of F-actin in growth cones from both groups (DIV1: $8.18 \pm 1.58 \mu \mathrm{m} / \mathrm{min}, n>20 / 3$, DAR1: $7.73 \pm 0.82 \mu \mathrm{m} / \mathrm{min}, n>50 / 3, p=0.80$; Fig. $4 E, F)$. Together, replating neither affected actin turnover nor retrograde $\mathrm{F}$-actin flow in growth cones.

\section{Growth cones from replated neurons respond normally to guidance cues}

Apart from studying actin dynamics, we tested whether growth cones from neurons of both groups respond differently to guidance cues. First, we determined growth cone size in phalloidin-stained DIV1 and DAR1 neurons on treatment with the neurotrophin brain-derived 
A

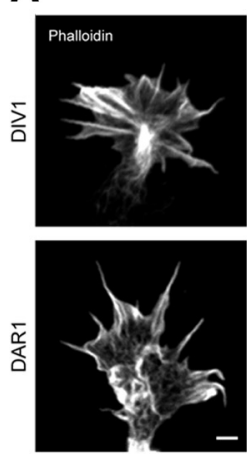

B

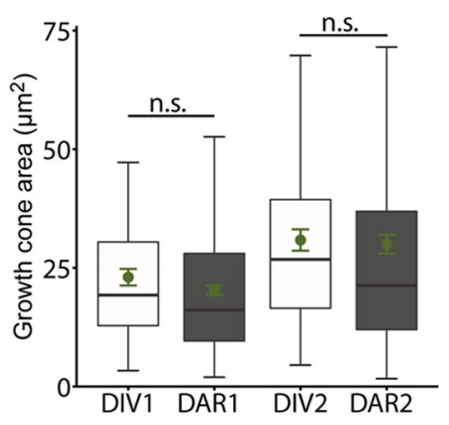

C

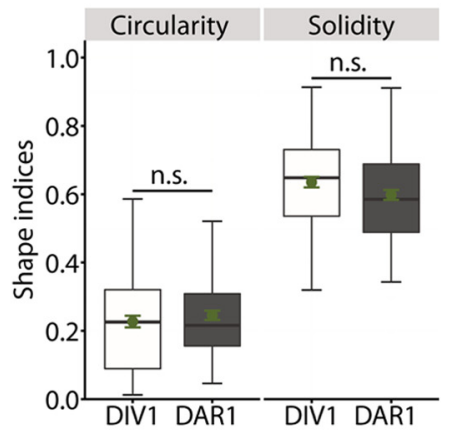

Figure 3. Replating does not alter growth cone size or morphology in hippocampal neurons. $\boldsymbol{A}$, Representative micrographs of phalloidin-labeled growth cones from non-replated and replated stage 2 neurons. $\boldsymbol{B}$, Growth cone size of non-replated and replated stage 2 neurons. C, Growth cone morphology (solidity, circularity) of non-replated and replated stage 2 neurons. Scale bar: $2 \mu \mathrm{m}$ $(\boldsymbol{A}) ; \mathrm{ns}: p>0.05$. Green dots indicate mean values with SEM.

neurotrophic factor (BDNF). As expected (Meier et al., 2011), BDNF increased growth cone size in non-replated neurons by $62 \%$ when compared with PBS-treated controls (PBS: $29.17 \pm 1.35 \mu \mathrm{m}^{2}$, BDNF: $47.13 \pm 2.40 \mu \mathrm{m}^{2}$, $p<0.001, n>130 / 3$; Fig. $5 A, B)$. BDNF similarly increased growth cone size in DAR1 neurons (PBS: $31.30 \pm 1.59$ $\mu \mathrm{m}^{2}$, BDNF: $\left.56.45 \pm 3.48 \mu \mathrm{m}^{2}, p<0.001, n>100 / 3\right)$. Hence, growth cones from DIV1 and DAR1 neurons respond similarly to BDNF.

Second, we investigated the effects of two different repellent cues, namely Ephrin A5 (EphA5) and Slit-1, on growth cones from non-replated and replated neurons (Meier et al., 2011; Ye et al., 2019). As a readout, we determined the fraction of collapsed growth cones in phalloidin-stained neurons on treatment with either EphA5 or Slit-1 and compared these fractions to PBS-treated control neurons (Fig. $5 C$ ). In agreement with normal growth cone morphology in replated neurons, the fraction of collapsed growth cones did not differ between DIV1 and DAR1 neurons before guidance cue treatment (DIV1: $20.71 \pm 2.15 \%$, DAR1: $20.20 \pm 2.19 \%, p=0.89, n>200 /$ 3; Fig. 5D). EphA5 and Slit-1 increased the fraction of collapsed growth cones roughly threefold in DIV1 neurons (EphA5: $60.95 \pm 2.59, p<0.001, n>300 / 3$; Slit-1: $53.67 \pm$ 3.17, $p<0.001, n>300 / 3)$. Similarly, both repellent cues strongly increased the fraction of collapsed growth cones in DAR1 neurons (EphA5: $58.80 \pm 6.26, p<0.001, n>210 / 3$; Slit-1: $50.80 \pm 4.04, p<0.001, n>200 / 3)$. Together, growth cones from non-replated and replated neurons respond similarly to the neurotrophin BDNF as well as the repellent cues EphA5 and Slit-1.

\section{Nucleofection-mediated gene inactivation allows to study early aspects of neuron differentiation in replated neurons}

The aforementioned approaches to test growth cone actin dynamics in replated neurons were based on nucleofection-based reporter gene expression. To extend our characterization of replated neurons to gene inactivation, we exploited primary hippocampal neurons from gene targeted mice (ADF-/-/Cfl $\left.1^{\mathrm{fl} / \mathrm{fl} \times}\right)$ lacking the actin- binding protein ADF and additionally carrying a floxed allele of the ADF homolog cofilin1 (Bellenchi et al., 2007). We chose this mouse model for a proof of concept, because actin-depolymerizing proteins of the ADF/cofilin family have been previously implicated in growth cone morphology (Gomez and Letourneau, 2014; Omotade et al., 2017), and because previous studies revealed redundant functions of ADF and cofilin1 in neurons (Zimmermann et al., 2015; Wolf et al., 2015; Flynn et al., 2012). To inactivate cofilin1, we electroporated ADF-/-/ $\mathrm{Cf} \mid 1^{\mathrm{fl} / f \mathrm{flx}}$ neurons before initial seeding with mCherry-tagged Cre recombinase (Cre), ADF-/-/Cfl $1^{\text {fl/ } / f \mid x}$ neurons expressing a catalytically inactive mCherry-Cre variant (Cre-mut) served as controls (Kullmann et al., 2020). We fixed Cre-expressing and Cre-mut-expressing ADF-/-/Cfl $1^{\mathrm{fl} / \mathrm{flx}}$ neurons at either DIV1 or DAR1 and determined growth cone size on phalloidin staining (Fig. 6A). At DIV1, we found that growth cone size in Cre-expressing ADF-/-/Cfl $1^{\text {fl/flx }}$ neurons was not different from Cre-mut-expressing controls (Cre-mut: $26.5 \pm 1.72 \mu \mathrm{m}^{2}$, Cre: $25.96 \pm 1.95 \mu \mathrm{m}^{2}$, $p=0.100, n>30 / 3$; Fig. $6 B$ ). Instead, growth cone size was strongly increased in Cre-expressing ADF-/ $/ / C f \mid 1^{f \mid x / f l x}$ neurons at DAR1 when compared with Cre-mut-expressing controls (Cre-mut: $24.40 \pm 2.2 \mu \mathrm{m}^{2}$, Cre: $48.50 \pm 3.74 \mu \mathrm{m}^{2}$, $p<0.001, n>80 / 3)$. Hence, ADF-/-/Cfl $1^{\text {fl/ } / f \mid x}$ neurons displayed the expected increase in growth cone size on genetic inactivation of ADF and cofilin1 at DAR1, but not at DIV1. Together, our replating protocol together with nucleofection-based gene inactivation before initial seeding allowed us to study the relevance of a gene of interest for early processes of neuron differentiation, thereby highlighting the utility of our approach.

\section{Discussion}

In the present study we report a protocol to reset DIV2 primary mouse hippocampus neurons into an undifferentiated stage. We combined replating with nucleofectionbased genetic manipulation (both reporter gene expression as well as gene inactivation by exploiting the Cre/ loxP system) before initial seeding of primary neurons. This approach allows a thorough analysis of neuron 

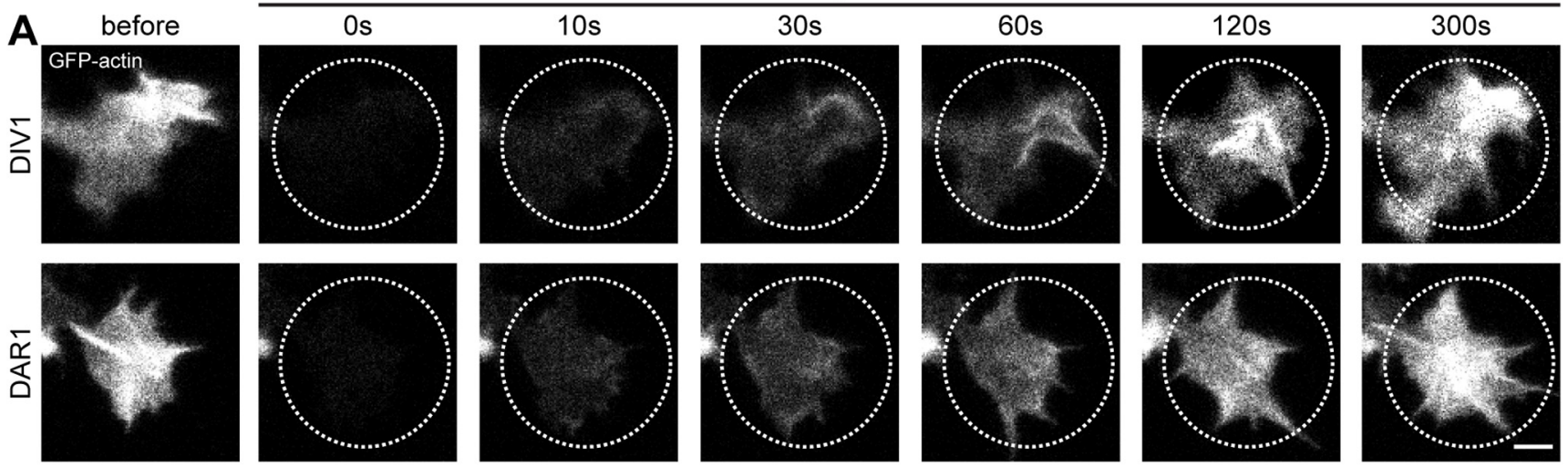

B

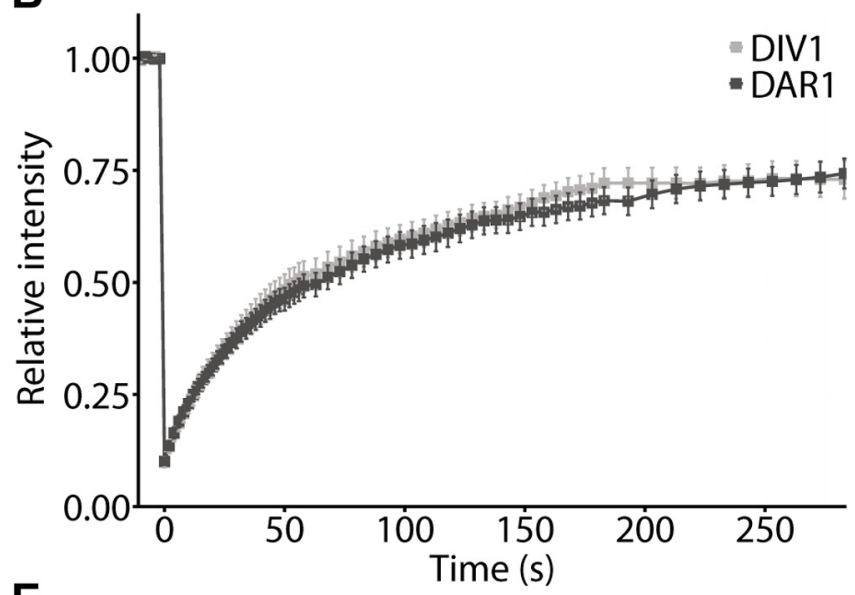

E
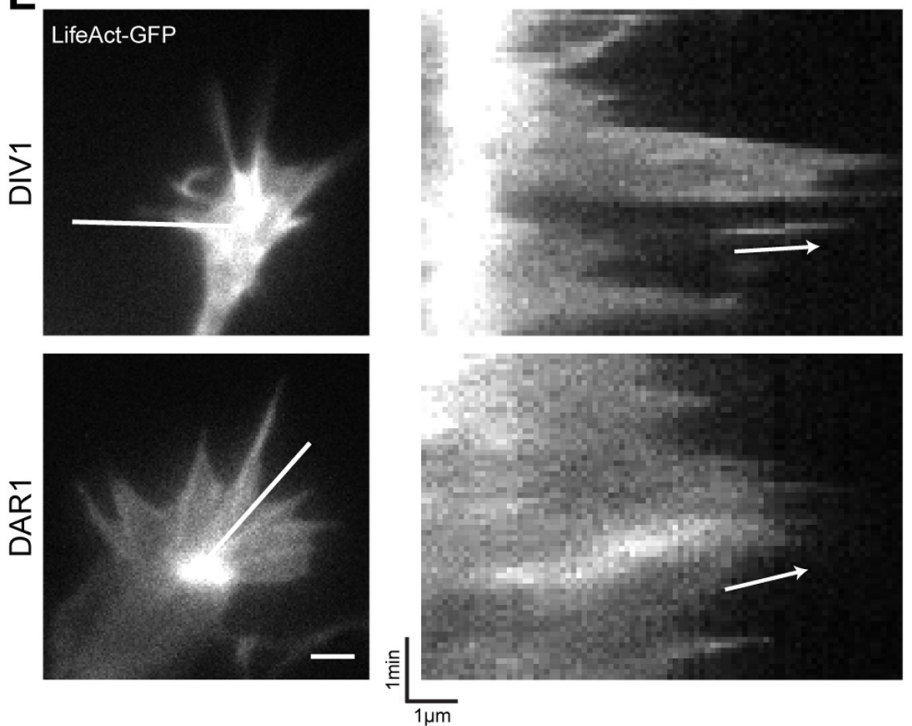

D

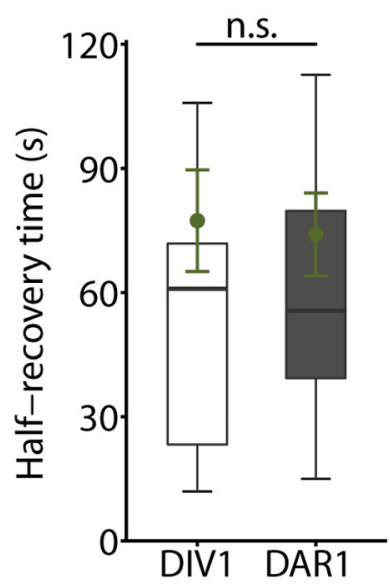

$\mathbf{F}$

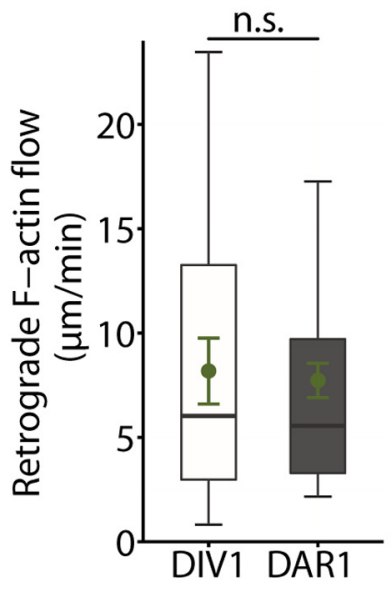

Figure 4. Replating does not impair actin dynamics in growth cones. $\boldsymbol{A}$, Image sequence of growth cones from GFP-actin-expressing non-replated and replated stage 2 neurons during FRAP analysis. $\boldsymbol{B}$, Recovery curves for GFP-actin in growth cones from stage 2 neurons at DIV1 and DAR1. C, Half-recovery time of GFP-actin in growth cones during FRAP experiment. $\boldsymbol{D}$, Stable actin fraction in growth cones during FRAP experiments. $\boldsymbol{E}$, Representative micrographs of growth cones from LifeAct-GFP-expressing non-replated and replated neurons. Lines indicate where kymographs (shown on the right) have been generated from. Arrows indicate the retrograde F-actin flow. $\boldsymbol{F}$, Velocity of retrograde F-actin flow in growth cones. Scale bars: $2 \mu \mathrm{m}(\boldsymbol{A}, \boldsymbol{D})$; ns: $p>0.05$. Green dots indicate mean values with SEM. 


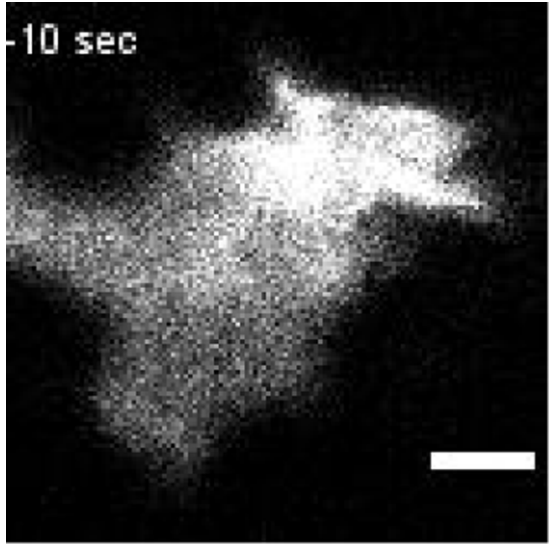

Movie 1. Movie showing GFP-actin recovery upon bleaching in the growth cone of a non-replated neuron at DIV1. Upon bleaching fluorescence recovery was recorded over a time course of $3 \mathrm{~min}$. Scale bar: $2 \mu \mathrm{m}$. [View online]

differentiation including early processes such as neurite formation and outgrowth or growth cone function.

Replating of cultured neurons has been reported for various neuron subtypes including primary dorsal root ganglia (DRG) neurons, primary cortical neurons or stem cell (SC)-derived neurons (Caviedes et al., 1990a,b; Koechling et al., 2011; Saijilafu et al., 2013; Frey et al., 2015; Biswas and Kalil, 2018; Calabrese et al., 2019; Lee et al., 2020a). Neuron replating has been implemented to reduce neuron complexity and cell membrane surface area, thereby improving accessibility for electrophysiological recordings, because passive membrane properties such as membrane capacitance or resistance were altered (Caviedes et al., 1990a,b). Further, it has been implemented to transfer SC-derived neurons from normal cell culture dishes onto 384 wells before experiments (Calabrese et al., 2019), and it has been exploited as a paradigm of axon regeneration (Saijilafu et al., 2013; Frey et al., 2015; Lee et al., 2020a). These studies differed in the procedure applied, and some of them only included a

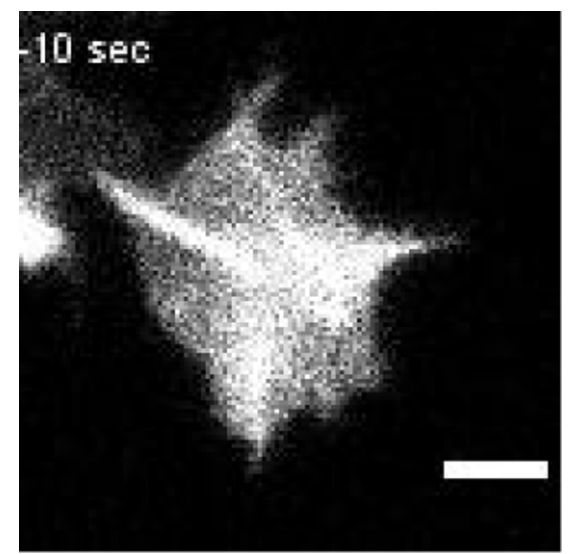

Movie 2. Movie showing GFP-actin recovery upon bleaching in the growth cone of a replated neuron at DAR1. Upon bleaching fluorescence recovery was recorded over a time course of 3 min. Scale bar: $2 \mu \mathrm{m}$. [View online]

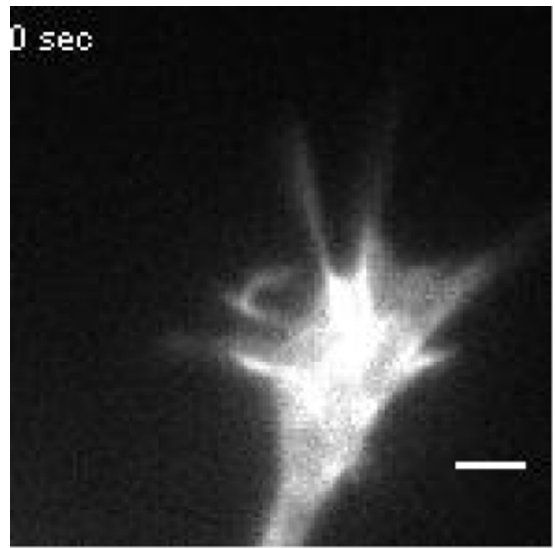

Movie 3. Movie showing a growth cone from a LifeAct-GFPtransfected non-replated neuron at DIV1. Images were acquired every $5 \mathrm{~s}$ for $5 \mathrm{~min}$. Scale bar: $2 \mu \mathrm{m}$. [View online]

brief and rather superficial description of the method. Moreover, these studies either did not focus on early aspects of neuron differentiation, did not systematically compare non-replated and replated neurons or did not combine replating with genetic manipulation. Hence, it remained unknown whether differentiation of replated neurons differed from non-replated neurons and whether a combination of genetic manipulation before initial seeding and replating allowed to study early aspects of neuron differentiation.

We compared cultured mouse hippocampal neurons that have been replated at DIV2 with non-replated neurons, focusing on early aspects of neuron differentiation up to 2 DAR. Our comparison included a categorization of neurons according to their differentiation stage as well as a thorough morphometric analysis. Neuron categorization did not reveal any differences between non-replated and replated neurons, thereby demonstrating that differentiation was largely preserved in replated neurons. Likewise, gross morphology was normal in replated neurons. However, they displayed some changes in neuron

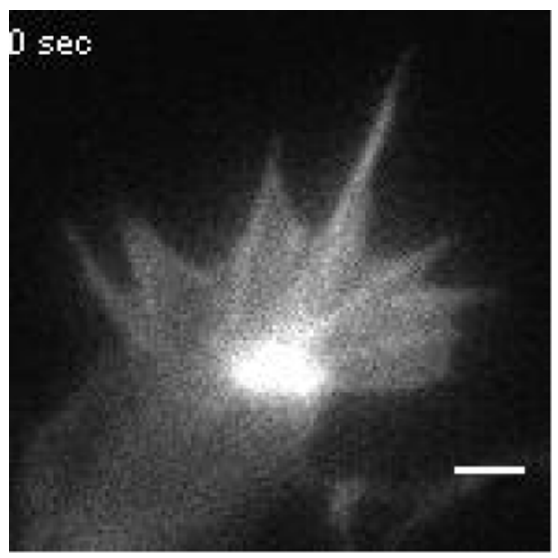

Movie 4. Movie showing a growth cone from a LifeAct-GFPtransfected replated neuron at DAR1. Images were acquired every $5 \mathrm{~s}$ for $5 \mathrm{~min}$. Scale bar: $2 \mu \mathrm{m}$. [View online] 

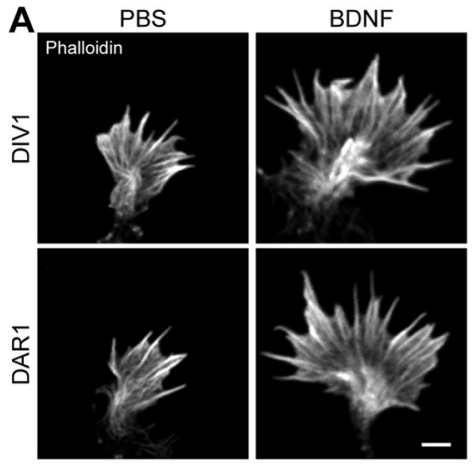

\section{B}
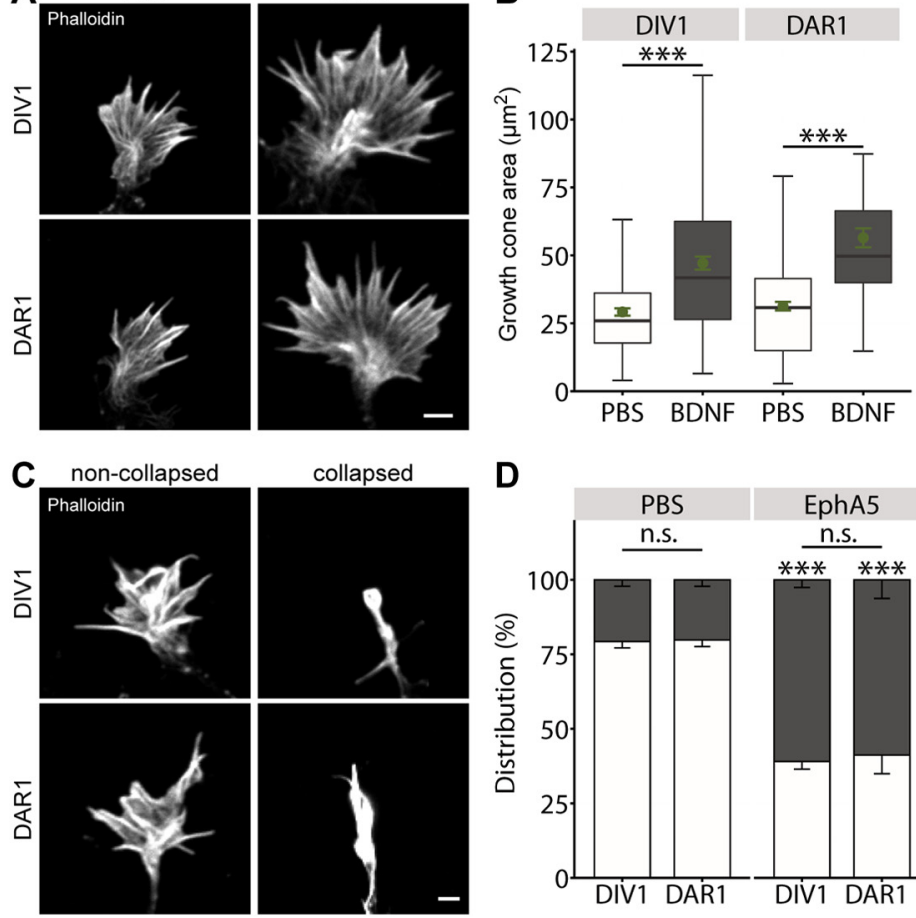

collapsed
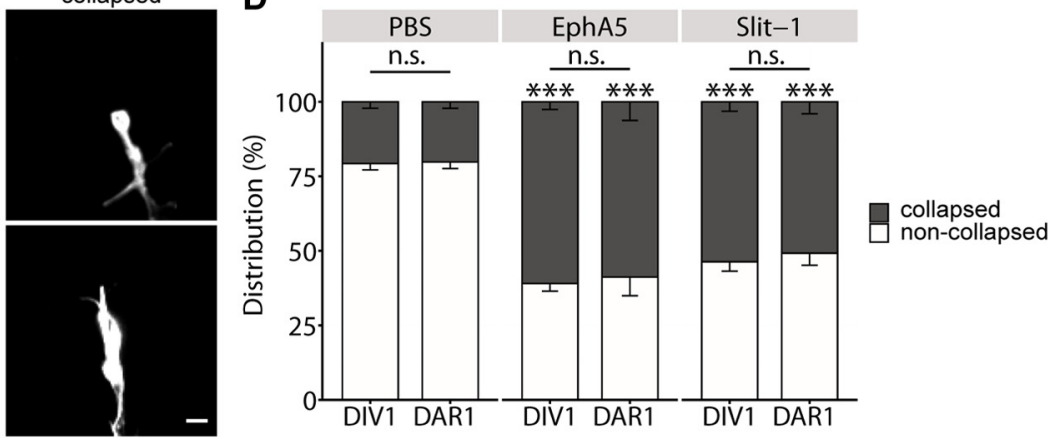

Figure 5. Normal response to guidance cues in growth cones from replated neurons. $\boldsymbol{A}$, Representative micrographs of phalloidinstained growth cones from non-replated and replated neurons treated with either PBS or BDNF. B, Growth cone size in non-replated and replated neurons treated with either PBS or BDNF. C, Representative micrographs of phalloidin-stained collapsed and non-collapsed growth cones from non-replated and replated neurons. $\boldsymbol{D}$, Fractions of collapsed and non-collapsed growth cones in non-replated and replated neurons before and after treatment with EphA5 and Slit-1. Scale bars: $2 \mu \mathrm{m}(\boldsymbol{A}, \boldsymbol{C}) ; n s: p>0.05$, ${ }^{\star \star \star} p<0.001$. Green dots in $\boldsymbol{A}$ indicate mean values with SEM.

morphology, which are likely not biologically relevant. Our data demonstrated that our replating procedure successfully reset DIV2 primary hippocampal neurons into an undifferentiated stage and that replated neurons differentiated very similar
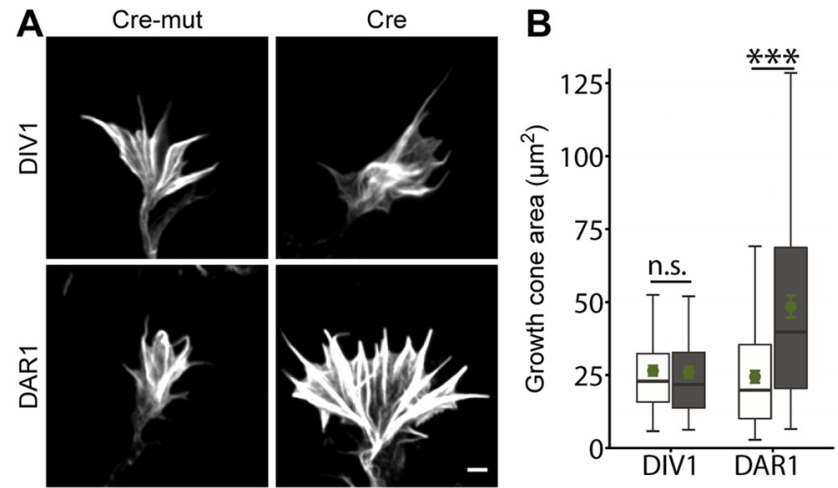

Figure 6. Replating allows studying the relevance of ADF/cofilin for early aspects of neuron differentiation. $\boldsymbol{A}$, Representative micrographs of phalloidin-stained growth cones from non-replated and replated ADF- $/-/ C f 1^{f|x / f| x}$ neurons expressing either Cre or Cre-mut. $\boldsymbol{B}$, Growth cone size in non-replated and replated ADF-/-/Cfl ${ }^{1 \mathrm{fl} / \mathrm{flx}}$ neurons expressing either Cre or Cre-mut. Scale bar: $2 \mu \mathrm{m}(\boldsymbol{A})$; ns: $p>0.05,{ }^{\star \star *} p<0.001$. Green dots indicate mean values with SEM. to non-replated neurons. Hence, replated neurons faithfully reflect normal differentiation of hippocampal neurons.

Further, we combined our replating procedure with nucleofection-based transfection of hippocampal neurons before initial seeding. We expressed reporter genes such as GFP-actin or LifeAct-GFP that allowed us to determine actin turnover as well as F-actin dynamics in growth cones as functional readouts. By FRAP analysis, we found that actin turnover in growth cones was not different between replated and non-replated neurons. Similarly, retrograde F-actin flow was unchanged in replated neurons. These finding demonstrated that our replating procedure did not alter actin dynamics in growth cones and let us suggest normal growth cone functions in replated neurons. Indeed, growth cones from replated neurons did not differ to those from non-replated neurons in their response to the neurotrophin BDNF or the repellent cues EphA5 and Slit-1. Together, our analysis in hippocampal neurons did not reveal any gross defects in differentiation, morphology or growth cone function in hippocampal neurons induced by the replating procedure. In contrast to our findings, a recent study revealed functional differences between non-replated and replated DRG neurons. Specifically, this study showed that axon regeneration occurred in replated adult DRG neurons even when gene transcription was inhibited by blocking RNA Polymerase II, while axon formation and 
outgrowth in non-replated adult DRG neurons required RNA Polymerase II activity (Saijilafu et al., 2013). However, it remained unknown whether such functional differences between replated and non-replated neurons is restricted to a specific cell types, i.e., adult DRG neurons, or whether these differences are present in all CNS and PNS neurons.

Apart from nucleofection of reporter genes, we exploited the Cre/loxP system to genetically remove actin-depolymerizing proteins of the ADF/cofilin family that have been previously linked to growth cone morphology (Gomez and Letourneau, 2014; Omotade et al., 2017). While growth cone size was unchanged in non-replated Cre-expressing ADF-/-/Cfl $1^{\mathrm{fl} / \mathrm{flx}}$ neurons at DIV1, it was strongly increased in replated Cre-expressing ADF-/-/Cfl $1^{\mathrm{fl} / \mathrm{flx}}$ neurons at DAR1. Differences in growth cone size between Cre-expressing ADF-/-/Cf1 $1^{\mathrm{fl} / \mathrm{fl} \mathrm{x}}$ neurons at DIV1 and DAR1 can be easily explained by the fact that DAR 1 neurons were $2 \mathrm{~d}$ longer in culture when compared with DIV1 neurons. Thus, DAR1 neurons had longer time to express Cre and to recombine the genome and, hence, to genetically remove cofilin 1 . In line with this, previous studies showed residual cofilin1 levels up to a few days on beginning of Cre expression in the mouse brain, but also in various cell types including isolated hippocampal neurons (Bellenchi et al., 2007; Rust et al., 2010; Flynn et al., 2012; Rehklau et al., 2012). Together, these data demonstrated that our replating protocol in combination with nucleofection-based gene inactivation allows us to study the relevance of a gene of interest for early aspects of neuron differentiation, different from nucleofected non-replated neurons. Hence, nucleofection combined with our replating protocol enables a more thorough analysis of neuron differentiation when compared with neurons that were nucleofected, but not replated.

In summary, we report a protocol to reset DIV2 primary mouse hippocampal neurons into an undifferentiated stage. This procedure is compatible with nucleofectionbased genetic manipulation of primary neurons before their initial seeding. Our approach allowed us (1) to express fluorescent reporters during neuron differentiation that are needed to address specific biological processes such as actin dynamics in growth cones or (2) to inactivate a gene of interest to study its function in early aspects of neuron differentiation. This approach is highly flexible, straightforward and far less labor-intensive and expensive than previous approaches, (1) in which transgenic mice such as Lifeact-expressing strains were exploited to study actin dynamics during early differentiation in cultured hippocampal neurons (Flynn et al., 2012) or (2) which required the breeding and scarification of a large number of knock-out mice and their control littermates. Hence, our replating protocol is very helpful to reduce the number of experimental animals, and it thereby complies with the $3 R$ principle for a more ethical use of animals in biomedical research (Russell and Burch, 1959; Lee et al., 2020b). While we here used expression of fluorescent reporters and Cre/loxP-based gene inactivation for a proof of principle, genetic manipulation can be easily expanded to gene silencing via RNA interference or other modes of gene deletion, e.g., by exploiting the CRISPR/Cas system. Taken together, a combination of nucleofection and replating of primary mouse hippocampal neurons is a powerful and versatile approach to comprehensively study the molecular mechanisms regulating neuron differentiation.

\section{References}

Bellenchi GC, Gurniak CB, Perlas E, Middei S, Ammassari-Teule M, Witke W (2007) N-cofilin is associated with neuronal migration disorders and cell cycle control in the cerebral cortex. Genes Dev 21:2347-2357.

Biswas S, Kalil K (2018) The microtubule-associated protein tau mediates the organization of microtubules and their dynamic exploration of actin-rich lamellipodia and filopodia of cortical growth cones. J Neurosci 38:291-307.

Calabrese B, Powers RM, Slepian AJ, Halpain S (2019) Post-differentiation replating of human pluripotent stem cell-derived neurons for high-content screening of neuritogenesis and synapse maturation. J Vis Exp (150). Advance online publication. Retrieved Aug 28, 2019. doi: $10.3791 / 59305$.

Caviedes P, Ault B, Rapoport SI (1990a) Replating improves whole cell voltage clamp recording of human fetal dorsal root ganglion neurons. J Neurosci Methods 35:57-61.

Caviedes P, Ault B, Rapoport SI (1990b) The role of altered sodium currents in action potential abnormalities of cultured dorsal root ganglion neurons from trisomy 21 (Down syndrome) human fetuses. Brain Res 510:229-236.

Chitsaz D, Morales D, Law C, Kania A (2015) An automated strategy for unbiased morphometric analyses and classifications of growth cones in vitro. PLoS One 10:e0140959.

da Silva JS, Dotti CG (2002) Breaking the neuronal sphere: regulation of the actin cytoskeleton in neuritogenesis. Nat Rev Neurosci 3:694-704.

Dos-Santos Carvalho S, Moreau MM, Hien YE, Garcia M, Aubailly N, Henderson DJ, Studer V, Sans N, Thoumine O, Montcouquiol M (2020) Vangl2 acts at the interface between actin and N-cadherin to modulate mammalian neuronal outgrowth. Elife 9:e51822.

Dotti CG, Sullivan CA, Banker GA (1988) The establishment of polarity by hippocampal neurons in culture. J Neurosci 8:1454-1468.

Dudek H, Ghosh A, Greenberg ME (2001) Calcium phosphate transfection of DNA into neurons in primary culture. Curr Protoc Neurosci Chapter 3:Unit 3.11.

Flynn KC, Hellal F, Neukirchen D, Jacob S, Tahirovic S, Dupraz S, Stern S, Garvalov BK, Gurniak C, Shaw AE, Meyn L, WedlichSöldner R, Bamburg JR, Small JV, Witke W, Bradke F (2012) ADF/ cofilin-mediated actin retrograde flow directs neurite formation in the developing brain. Neuron 76:1091-1107.

Frey E, Valakh V, Karney-Grobe S, Shi Y, Milbrandt J, DiAntonio A (2015) An in vitro assay to study induction of the regenerative state in sensory neurons. Exp Neurol 263:350-363.

Gomez TM, Letourneau PC (2014) Actin dynamics in growth cone motility and navigation. J Neurochem 129:221-234.

Koechling T, Khalique H, Sundström E, Ávila J, Lim F (2011) A culture model for neurite regeneration of human spinal cord neurons. $J$ Neurosci Methods 201:346-354.

Kullmann JA, Trivedi N, Howell D, Laumonnerie C, Nguyen V, Banerjee SS, Stabley DR, Shirinifard A, Rowitch DH, Solecki DJ (2020) Oxygen tension and the VHL-Hif1 $\alpha$ pathway determine onset of neuronal polarization and cerebellar germinal zone exit. Neuron 106:607-623.

Lee J, Shin JE, Lee B, Kim H, Jeon Y, Ahn SH, Chi SW, Cho Y (2020a) The stem cell marker Prom1 promotes axon regeneration by down-regulating cholesterol synthesis via Smad signaling. Proc Natl Acad Sci USA 117:15955-15966.

Lee KH, Lee DW, Kang BC (2020b) The ' $R$ ' principles in laboratory animal experiments. Lab Anim Res 36:45. 
Meier C, Anastasiadou S, Knöll B (2011) Ephrin-A5 suppresses neurotrophin evoked neuronal motility, ERK activation and gene expression. PLoS One 6:e26089.

Müller B, Stahl B, Bonhoeffer F (1990) In vitro experiments on axonal guidance and growth-cone collapse. J Exp Biol 153:29-46.

Ohki EC, Tilkins ML, Ciccarone VC, Price PJ (2001) Improving the transfection efficiency of post-mitotic neurons. J Neurosci Methods 112:95-99.

Omotade OF, Pollitt SL, Zheng JQ (2017) Actin-based growth cone motility and guidance. Mol Cell Neurosci 84:4-10.

Rehklau K, Gurniak CB, Conrad M, Friauf E, Ott M, Rust MB (2012) ADF/cofilin proteins translocate to mitochondria during apoptosis but are not generally required for cell death signaling. Cell Death Differ 19:958-967.

Riedl J, Crevenna AH, Kessenbrock K, Yu JH, Neukirchen D, Bista M, Bradke F, Jenne D, Holak TA, Werb Z, Sixt M, Wedlich-Soldner R (2008) Lifeact: a versatile marker to visualize F-actin. Nat Methods 5:605-607.

Russell WMS, Burch RL (1959) The principles of humane experimental technique. London: Methuen and Co.

Rust MB, Gurniak CB, Renner M, Vara H, Morando L, Görlich A, Sassoè-Pognetto $M$, Banchaabouchi MA, Giustetto $M$, Triller A, Choquet D, Witke W (2010) Learning, AMPA receptor mobility and synaptic plasticity depend on n-cofilin-mediated actin dynamics. EMBO J 29:1889-1902.

Saijilafu, Hur EM, Liu CM, Jiao Z, Xu WL, Zhou FQ (2013) PI3KGSK3 signalling regulates mammalian axon regeneration by inducing the expression of Smad1. Nat Commun 4:2690.
Schindelin J, Arganda-Carreras I, Frise E, Kaynig V, Longair M, Pietzsch T, Preibisch S, Rueden C, Saalfeld S, Schmid B, Tinevez JY, White DJ, Hartenstein V, Eliceiri K, Tomancak P, Cardona A (2012) Fiji: an open-source platform for biological-image analysis. Nat Methods 9:676-682.

Sun M, Bernard LP, Dibona VL, Wu Q, Zhang H (2013) Calcium phosphate transfection of primary hippocampal neurons. J Vis Exp 81: e50808.

Viesselmann C, Ballweg J, Lumbard D, Dent EW (2011) Nucleofection and primary culture of embryonic mouse hippocampal and cortical neurons. J Vis Exp 47:2373.

Wolf M, Zimmermann AM, Görlich A, Gurniak CB, SassoèPognetto M, Friauf E, Witke W, Rust MB (2015) ADF/cofilin controls synaptic actin dynamics and regulates synaptic vesicle mobilization and exocytosis. Cereb Cortex 25:28632875.

Ye X, Qiu Y, Gao Y, Wan D, Zhu H (2019) A subtle network mediating axon guidance: intrinsic dynamic structure of growth cone, attractive and repulsive molecular cues, and the intermediate role of signaling pathways. Neural Plast 2019:1719829.

Zeitelhofer M, Vessey JP, Thomas S, Kiebler M, Dahm R (2009) Transfection of cultured primary neurons via nucleofection. Curr Protoc Neurosci Chapter 4:Unit 4.32.

Zimmermann AM, Jene T, Wolf M, Görlich A, Gurniak CB, SassoèPognetto M, Witke W, Friauf E, Rust MB (2015) Attention-deficit/ hyperactivity disorder-like phenotype in a mouse model with impaired actin dynamics. Biol Psychiatry 78:95-106. 